\title{
Data mining algorithms for land cover change detection: a review
}

\author{
SANGRAM PANIGRAHI $^{1, *}$, KESARI VERMA $^{1}$ and PRIYANKA TRIPATHI ${ }^{2}$ \\ ${ }^{1}$ Department of Computer Applications, National Institute of Technology Raipur, Raipur 492010, India \\ ${ }^{2}$ Department of Computer Engineering and Applications, National Institute of Technical Teachers' Training and \\ Research, Bhopal 462002, India \\ e-mail: sangrampanigrahi.sp@gmail.com; kverma.mca@nitrr.ac.in; ptripathi@nitttrbpl.ac.in
}

MS received 16 March 2016; revised 2 March 2017; accepted 4 March 2017; published online 24 November 2017

\begin{abstract}
Land cover change detection has been a topic of active research in the remote sensing community. Due to enormous amount of data available from satellites, it has attracted the attention of data mining researchers to search a new direction for solution. The Terra Moderate Resolution Imaging Spectrometer (MODIS) vegetation index (EVI/NDVI) data products are used for land cover change detection. These data products are associated with various challenges such as seasonality of data, spatio-temporal correlation, missing values, poor quality measurement, high resolution and high dimensional data. The land cover change detection has often been performed by comparing two or more satellite snapshot images acquired on different dates. The image comparison techniques have a number of limitations. The data mining technique addresses many challenges such as missing value and poor quality measurements present in the data set, by performing the preprocessing of data. Furthermore, the data mining approaches are capable of handling large data sets and also use some of the inherent characteristics of spatio-temporal data; hence, they can be applied to increasingly immense data set. This paper stretches in detail various data mining algorithms for land cover change detection and each algorithm's advantages and limitations. Also, an empirical study of some existing land cover change detection algorithms and results have been presented in this paper.
\end{abstract}

Keywords. Data mining; land cover change detection algorithm; time series data; high dimensional data.

\section{Introduction}

The land cover change impacts on local climate, hydrology, radiation and bio-geochemistry. Studies have shown that deforestation has a significant impact on local weather, greenhouse gas emissions in the atmosphere (carbon dioxide), cloudiness and rainfall, etc. The quantifiable knowledge about changes that occur in land cover and land use at a global scale is important to make effective planning for conservation and sustainable use of natural resources such as forest cover and agricultural land. Due to the importance of land cover and land use change detection, it has been a topic of active research in the remote sensing community. The land cover change detection problem is to detect when the land cover of a particular location has been converted from one type to another, i.e. conversion of forested land to barren land due to fires, droughts, insect damage, logging, etc.

Land cover change detection has often been carried out by employing image-based techniques, comparing two or

*For correspondence more satellite snapshot images acquired on different dates [1]. In the image-based techniques, the accuracy of result solely depends on the domain scientist experience. It does not automatically provide an idea about the rate of change, i.e., whether sudden change or gradual change occurs in a particular location. The image-based techniques compare only between two satellite snapshot images acquired on different dates and do not consider the information of these dates; hence, an actual date of change is difficult to find. These techniques could not derive information about ongoing landscape processes; also, they have been focused on relatively small areas only. The changes that occur outside the image acquisition windows are not mapped and these techniques are also unsuitable for global scale application [2].

The time series data addresses to overcome the aforementioned problem with image-based techniques [3] or image-based data [4]. Recently, approaches based on data mining or time series have been employed to detect land cover changes $[5,6]$. The data mining technique applied on time series data has significant advantages over the comparison of snapshot images on selected dates. In this technique, detection of changes is based on the pattern of spectral response of the landscape over time rather than the 
differences between two or more images collected on different dates. The data mining technique also addresses the time of change, type of change, the extent of change, pattern of re-growth, etc. [2].

"There are broadly three types of vegetation changes in time-series-based data: sudden change, gradual change and land cover type change. Sudden change is abrupt and unexpected reduction in large vegetation index that extends over multiple months (greater than seasonal months) or multiple years at the time. The sudden change is also called abrupt change. It occurs in vegetation index time series due to events like a forest fire, flood, mechanized clearings, etc. The gradual change is a gradual increase or decrease in vegetation trends spanning over multiple years in time. The gradual changes represent plantation, forest degradation such as that due to long-term droughts, beetle infestations, gradual logging, etc. The land cover type change identifies any change in the vegetation type such as a change from one land cover to another or change in cropping patterns, clearing of forests for agriculture, urban expansion, etc." [2]. In this paper, we discuss briefly about various existing data mining approaches for land cover change detection, using time series data. The methodology for land cover

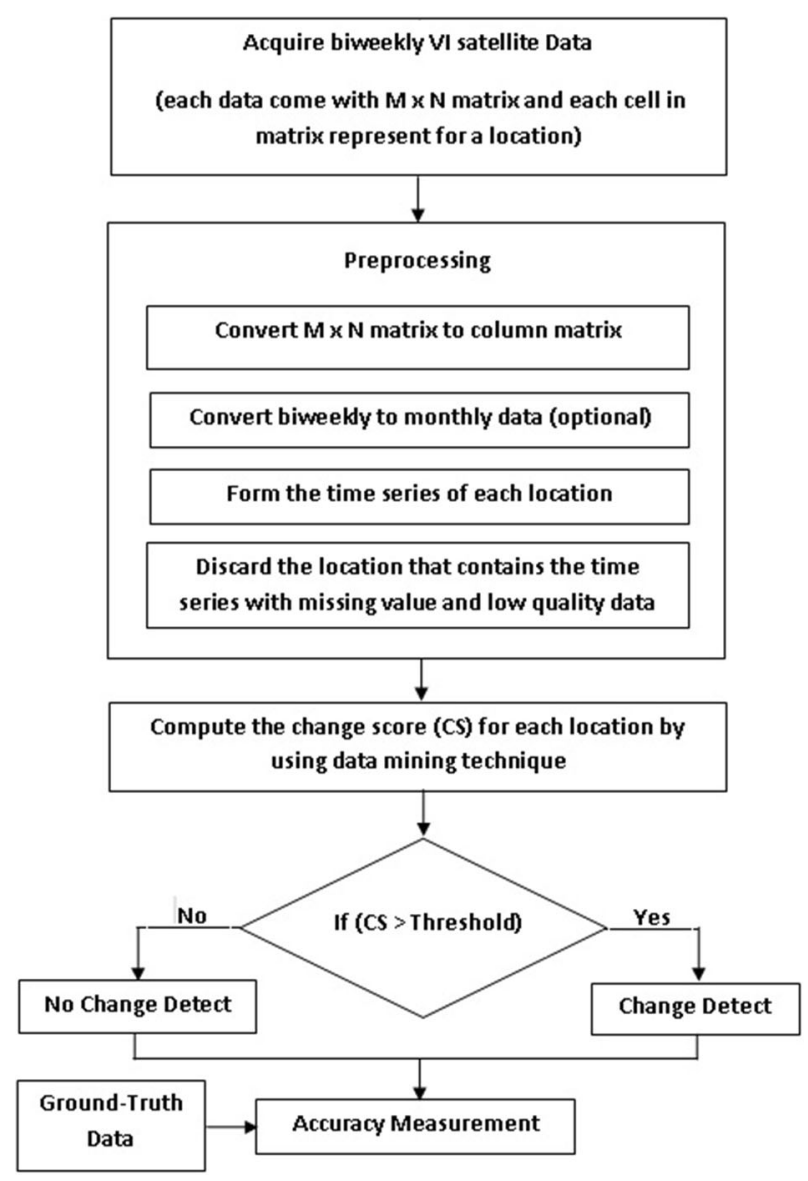

Figure 1. Methodology of land cover change detection using data mining. change detection using the data mining technique has been shown in figure 1 .

The rest of the paper is organized as follows. Section 2 briefly describes about the data sets used for land cover change detection. The various existing data mining approaches for land cover change detection are explained; also, advantages and limitations of each algorithm are discussed in section 3. An empirical study of some existing change detection algorithms is carried out and its results are discussed in section 4 . Finally, we discuss and conclude the paper in sections 5 and 6 , respectively.

\section{Moderate resolution imaging spectroradiometer vegetation indices}

For mapping land cover, the remote-sensing-based techniques offer the most cost-effective data. The global coverage with large amounts (i.e., terabytes) of temporal, spatial and spatio-temporal data has been generated by NASA's earth observation satellites, which are freely available for research work. The quantity of the greenness of vegetation at a particular location is expressed by Vegetation Indices (VIs), which are an important ecosystem variable. The VI is used for phonologic monitoring; biophysical derivation of radiometric and structural vegetation parameters; classification of vegetation and land cover; detection of drought, deforestation, land degradation, land change and monitoring of detecting land change [7]. The VI ranges between 0 and 1 . The VI from 0 to 0.1 designates barren areas of rock, sand or snow; values ranging between 0.2 and 0.3 correspond to shrub and grassland; high values between 0.6 and 0.9 represent temperate and tropical rainforests. In general, for land cover change detection, two global vegetation indices, NDVI and EVI are used. After launching of Moderate Resolution Imaging Spectroradiometer (MODIS) Terra and Aqua satellite by NASA, the EVI is available from year 2000 to date. The NDVI, which is available from 1981 to date, from the NOAA-AVHRR series, has been extended by MODIS. The EVI and NDVI data have been provided in the HDF-EOS file format by MODIS Land Discipline Group with varying spatial (250 $\mathrm{m}, 500 \mathrm{~m}, 1 \mathrm{~km}$ and $5600 \mathrm{~m}$ or $0.05^{\circ}$ ) and temporal (16day, monthly) resolutions. The data range in the HDF-EOS file is stored from -2000 to +10000 with a fill value of -3000 . To convert data range between 0 and 1 , first the negative data values have been discarded and then the each data value is divided by 10,000 in the preprocessing phase. These data sets also include quality assurance information, which addresses under which conditions each pixel was acquired and processed [8]. The NDVI and EVI composite data products are available for public download at the Land Processes Distributed Active Archive Center (LP DAAC) [Available: https://www.lpdaac.usgs.gov/products/modis_ products_table]. 


\section{Review of algorithms}

This section briefly discusses various data mining approaches for land cover change detection. Furthermore, among these, some approaches have the ability to detect sudden changes, some have the ability to detect gradual changes and few are capable of finding both changes in vegetation index time series data. In addition, each algorithm's limitation and advantages have been discussed over in detail. For each location, the vegetation index time series is an input to the approach and as a result the change score corresponding to that location is obtained. The location with change score greater than the predefined threshold value is called change. These techniques are able to take advantage of the inherent structure present in vegetation index time series data, i.e., seasonality. The general notations, which are used in this paper for time series change detection, are shown in table 1.

\subsection{Modified Lunetta algorithm}

Lunetta and his team [9] proposed this technique and applied to NDVI time series data of North Carolina, to identify the land cover change. The Lunetta technique first computes the annual sum and then finds the difference between consecutive annual sums to detect whether a change has occurred between the two years, $t_{i}$ and $t_{i+1}$. The calculated results are equivalent to applying first-order differencing to the time series of annual totals [10]. The resulting differences are accompanied by an approximate normal distribution with $\mu=0$, when no change has taken place. Finally the $z$-score of each annual sum of differences is computed by determining the standard deviation through considering all the spatial neighbours of the pixel (i.e. location) present in that time window. Thus, the Lunetta algorithm is a spatio-temporal change detection technique. A particular location is considered as change if the $z$-score is greater than the threshold $(\tau)$ value and indicates that a change has been taking place between the consecutive years corresponding to $t_{i}$ and $t_{i+1}$.

Table 1. Notations for time series change detection [2].

\begin{tabular}{lc}
\hline Data set & $D$ \\
Land location & $N$ \\
Length of time series (EVI/NDVI) & $T$ \\
Trailing month & $T M \leftarrow T \% 12$ \\
Number of complete years in time series $T$ & $y \leftarrow \frac{T-T M}{12}$ \\
The total number of months in y years & $t \leftarrow y * 12$ \\
The time series with $t$ number of months & $A$ \\
The seasonal length or number of time steps & $p=23$ for \\
$\quad$ corresponding to one year of data & biweekly data \\
& $p=12$ for \\
& monthly data \\
\hline
\end{tabular}

In order to study land cover change detection problem, Boriah and his team [11] have slightly modified the Lunetta algorithm [9], which is called a Modified Lunetta algorithm. The time series for each location is processed by the Modified Lunetta algorithm as follows.

Step 1 . The annual sum of EVI, $a_{j}$, is computed

$$
a_{j} \leftarrow \sum_{t=1}^{12} A_{t}
$$

where $j$ represents the year, which varies from 1 to $y . A_{t}$ is the EVI value at time step $t$.

Step 2. The difference between annual sums of two consecutive years, $d_{j}$, is computed, where $j$ varies from 1 to $y-1$.

$$
d_{j} \leftarrow a_{j+1}-a_{j} .
$$

Step 3. The $Z$-score value, $Z_{i}$, is computed for each $d_{j}$ by subtracting the mean and standard deviation of $d_{j}$. Here, $i$ and $j$ vary from 1 to $y-1$. Let $Z_{1}, Z_{2}, \ldots, Z_{y-1}$ be the $Z$ score values corresponding to $d_{1}, d_{2}, \ldots, d_{y-1}$, respectively.

Step 4 . The change score for a time series can be assigned by taking the maximum of the absolute value of $Z_{1}, Z_{2}, \ldots, Z_{y-1}$.

$$
C S \leftarrow \max \left\{a b s\left\{Z_{i}\right\}\right\}
$$

This algorithm has low computational complexity and is highly scalable; it is also less affected by noise. The algorithm has ability to detect both increase and decrease in vegetation. It has detected single change, but could detect multiple changes by small modification. The Lunetta algorithm considers the spatial neighbourhoods of the pixel, which account for time periods that have high natural variability or variability induced by noise. However, the Modified Lunetta algorithm does not include the spatial neighbourhoods of the pixel.

This algorithm fails to deal with multiple missing values present in input time series. For computation of change score of a particular location, this algorithm converts the time series into annual sums; thus, the estimated time of change is precise only up to the year of change. The spatial neighbourhoods included by this algorithm should be coherent, but practically land covers may be non-coherent in nature because the data set may consist of multiple land cover types.

The computational complexity and storage requirement of the Modified Lunetta algorithm are $\mathrm{O}(N t)$ and $\mathrm{O}(N)$, respectively [2]. Here, $N$ is the number of locations and $t$ is the time required to process the single location, i.e., $\mathrm{O}(t)$.

\subsection{CUSUM MEAN algorithm}

Cumulative sum (CUSUM) algorithm was originally developed for the domain of process control [12] and is a 
well-known change detection algorithm. CUSUM technique is a parameter change technique that uses the mean of the observations as a parameter to identify the dispersion of the data values. The basic CUSUM scheme has an expected value $(\mu)$ for the process. This technique maintains a running statistic, the cumulative sums (CSs) of deviations, by obtaining the difference of every observation to the expected value. If CS is approximately 0 , there is no change in the time series. A time series is flagged as change if CS is greater than a user-specified threshold. The CS with high positive or low negative value, respectively, implies increase or decrease in the mean value of vegetation.

For land cover change detection, Kucera and his team [13] proposed a robust method to compute the change score for CUSUM technique. In this technique, the expected value $\mu$ is calculated by taking the mean of all observations. Subsequently, cumulative sums, $S_{i}$, are computed; later its maximum $\left(S_{\max }\right)$ and minimum $\left(S_{\min }\right)$ values are found [14]. Then the $S_{\text {diff }}$ is computed as $S_{\text {diff }}=\left(S_{\max }-S_{\min }\right) / 2$. To determine the confidence of $S_{\text {diff }}$, a bootstrap procedure is employed, which determines explicitly the degree to which such a score can occur by chance. The $S_{\text {diff_b }}$ is obtained by randomly reordering the input data in the bootstrap procedure. The confidence level computed by number of $S_{\text {diff }}$ will be substantially higher than $S_{\text {diff_b }}$, when the input data are reordered over time $(N)$, i.e. $\left|S_{\text {diff }}>S_{\text {diff_b }}\right| / N$. On the other hand, there will be no substantial conflicts between these two differences, i.e., CUSUM charts for original and bootstrapped time series will look similar, if the time series is stable over time [2].

Boriah and his team [15] altered the basic CUSUM algorithm and it is called as CUSUM MEAN. The time series for each location is processed by the CUSUM MEAN algorithm as follows.

Step 1. The algorithm has an expected value, set as incontrol mean $\mu$, which is computed by taking the average of the first annual cycle of the time series.

$$
\mu \leftarrow \frac{\sum_{t=1}^{12} A_{t}}{12}
$$

where $A_{t}$ is the EVI value at time step $t$.

Step 2. The CUSUM, $C S_{i}$ for each time step present in time series is computed as follows:

$$
\begin{aligned}
& \text { initialize, } C S_{0} \leftarrow 0 \\
& C S_{i} \leftarrow C S_{i-1}+\left(A_{t}-\mu\right)
\end{aligned}
$$

where both $i$ and $t$ vary from 1 to $t$.

Step 3. $C S_{\min }$ and $C S_{\max }$ values are calculated from the CUSUM series. The $C S_{\min }$ and $C S_{\max }$ are minimum and maximum CUSUM values, respectively, in the CUSUM series:

$$
\begin{aligned}
C S_{\min } & \leftarrow \min \left(C S_{i}\right) \\
C S_{\max } & \leftarrow \max \left(C S_{i}\right) .
\end{aligned}
$$

Step 4. If the absolute value of $C S_{\max }$ is greater than the absolute value of $C S_{\min }$, then set $C S_{\max }$ value as change score and the resultant change type as positive. Otherwise, set the $C S_{\min }$ value as change score and the resultant change type as negative.

The CUSUM-based techniques are used for detecting small and gradual changes. The CUSUM technique is fast because of simple methods of score calculation; also, it is highly scalable. It also detects the time of change close to the actual change with accuracy up to the temporal resolution of the input data set.

The algorithm is highly sensitive to noise and missing data. Multiple changes occurring in time series are not detected by this technique; it also has inability in detecting changes in both directions simultaneously. This method does not provide the period of time change. The algorithm is sensitive to small pattern changes in the periodic cycle that do not represent real changes.

The computational complexity and storage requirement of the CUSUM MEAN are $\mathrm{O}(N t)$ and $\mathrm{O}(N)$, respectively [2]. There are $N$ locations and computational time for a single location is $\mathrm{O}(t)$.

\subsection{Clustering-based land cover change detection}

Boriah and his team [16] and C. Potter and his team [17] used the $k$-mean algorithm [18] to cluster the 250-m EVI time series to produce five number of distinct clusters with centroids. For the land cover change detection problem, Boriah and his team [16] discussed two clustering-based techniques on EVI data, one is the Distance to Cluster Centroid and another is the Confidence Intervals Around Cluster Centroids. The Distance to Cluster Centroid scheme is based on the assumption that if the majority of time series in a data set does not exhibit a change, at that time some outliers in such a data set will be change points. The method proceeds as follows: the points are first clustered in $k$ major classes. Subsequently the $L 1$ (Manhattan) distance is computed from each point to its nearest centroid. Finally, the outliers are extracted based on this distance. Since clustering is the central component of this scheme, the results quality normally depends on the clustering quality. The Confidence Intervals Around Cluster Centroids is based on the assumption that most of the data can be grouped into well-defined clusters and that the majority of data does not exhibit a change. The method proceeds as follows: the data (the time series for location) are clustered into $k$ major classes. Further, for each cluster, the confidence interval is constructed by an upper and lower limit based, respectively, on the 5th and 95th percentile from the cluster centroid. The numerical score is assigned to a location by counting the number of data points (months) that lie outside the interval. Data points are characterized as change points (or outliers) based on their relationship to the confidence intervals. Among the outliers, a data point with 
high value is considered as change point of the time series. The time series for each location is processed as follows.

Step 1. Randomly select the $k$ points (time series) as initial centroids of $k$-cluster from data set $D$.

Step 2. Compute the $L 1$ (Manhattan) distance of each point in the data set from each of the centroids and assign each point to its closest centroid.

Step 3. Recompute the centroids of each cluster by calculating the means of the attribute values of the points present in each cluster.

Step 4. Repeat steps 2 and 3 until the centroids do not change or change very little.

Step 5. For each cluster, confidence interval is constructed by an upper and lower limit based, respectively, on the 5th and 95th percentile from the cluster centroid.

Step 6. The score is assigned to a location by counting the number of data points (months) lies outside the interval.

Salmon and his team [19] proposed an automated change detection algorithm for MODIS 8 days composite surface reflectance data. A subsequence $x_{p}(t)$ was selected using temporal sliding window techniques from a given time series data $x(t)$ of length $N$. Later, Fast Fourier Transform (FFT) of $x_{p}(t)$ was computed, i.e., $X_{p}(f)=\left|F\left(x_{p}(t)\right)\right|$, which decomposes the time sequence values into components of different frequencies with phase offsets. Since the time series $x_{p}(t)$ was annually periodic, this was translated into frequency components in the frequency spectrum, which have fixed positions. It reduced the number of features needed to represent the feature space and thus reduced the dimensionality. Later, three different clustering techniques were applied: Ward [20], $K$-mean [18] and Expectation - Maximization (EM) [21] on these extracted features, $X_{p}(f)$. Land cover change is declared when consecutive subsequences that are extracted from one MODIS time series transitions from one cluster to another cluster remains in the newly assigned cluster for the rest of the time series [19].

One of the strengths of this scheme is that it is robust to noisy observations that are interspersed throughout the time series. The drawback of the distance to centroid scheme is that there is a heavy dependence on the cluster quality. If there is a group of points that have similar time series but are not in a cluster by themselves, then these points may be discovered as change points. This algorithm has high computational complexity, primarily because the initial cluster/model generation step is typically a difficult step. In addition, the clusters must be fine-tuned with a post-processing step to ensure that noise clusters are removed. This algorithm did not explore the seasonality components in the data.

The computational complexity of the $K$-mean algorithm is $\mathrm{O}(K N T)$, the total number of operations for an iteration. Assuming that the algorithm converges after $I$ iterations, the total number of operations for the algorithm is
$\mathrm{O}(I K N T)$. The storage required is $\mathrm{O}((N+K) T)$, where $N$ is the number of locations and $T$ is the length of the time series in data set $D$.

\subsection{Recursive Merging algorithm}

Boriah and his team [22, 23] proposed a segmentationbased algorithm, which splits up the time series into two pieces and each is called as a segment. The two segments separated by common boundary is called the change point, which provides the time of change. In order to detect land cover change of a particular location, this algorithm employs seasonality. At a particular location, if the seasonal cycle is very similar between two consecutive years in the time series, the change score is nearly 0 and there is no change in land location; otherwise, based on the degree of the seasonal difference, the change score is assigned. The Recursive Merging algorithm is based on bottom-up approach [10]. This algorithm proceeds by merging the two annual segments, which are similar as well as consecutive in time. The merging cost has been calculated by computing the Manhattan distance between the corresponding months in annual segments. The central idea of this algorithm is that similar annual cycles that are consecutive in time present in the time series are merged and then merging cost is calculated recursively. The maximum value of final merging cost corresponding to change occurs in the time series and vice-versa. The details of the processing of each time series are as follows.

Step 1. Let $a_{j}$ be the list of annual cycles, which varies from 1 to $y$, such that the list of annual cycles is $a_{1}, a_{2}, \ldots, a_{y}$, where $a_{j}=A_{(t-S+1), \ldots, t}, A_{t}$ is the EVI value at time step $t$; here, $t=12 j$ and $S=12$. This algorithm first find outs annual distances between two consecutive years, $d_{i}$, where $i$ varies from 1 to $y-1$.

$$
d_{i} \leftarrow A_{(t-S+1), \ldots, t}-A_{(t+1), \ldots,(t+s)} .
$$

Step 2. Merge the two most similar annual cycles and prepare the new list, $a_{j}$ with one less annual cycle. The distance between two merged cycles has been stored. The similarity is decided on consecutive annual cycles with minimum distance. The similarity between two consecutive annual cycles can be found at the initial, middle and end portions of the list. Suppose $a_{1}$ and $a_{2}$ are the two most similar annual cycles in the initial portion of the list; then, at the end of the step, form the new list with one less element, $\left\{\frac{a_{1}+a_{2}}{2}, a_{3}, \ldots, a_{y}\right\}$, along with the distance, $s_{1} \leftarrow\left(A_{1, \ldots, 12}-A_{13, \ldots, 24}\right)$.

Step 3. Recursively apply the steps 1 and 2 until one element is left in the list. Finally, the distance score result $\left(s_{k}\right)$ is obtained, where $k$ varies from 1 to $y-1$, such that $s \leftarrow\left\{s_{1}, s_{2}, \ldots, s_{y-1}\right\}$.

Step 4. Further, the change score $(C S)$ is computed by taking the ratio of score $_{\max }$ to score $_{\text {min }}$. 


$$
\begin{gathered}
\text { score }_{\text {min }} \leftarrow \min (s) \\
\text { score }_{\text {max }} \leftarrow \max (s) \\
C S \leftarrow\left\{\begin{array}{lc}
\frac{\text { score }_{\text {max }}}{\text { score }_{\text {min }}} & \text { if } \text { score }_{\text {min }} \neq 0 \\
\frac{\text { score }_{\text {max }}}{\text { score }_{\text {min }}+\epsilon} & \text { otherwise, here } \epsilon \neq 0 .
\end{array}\right.
\end{gathered}
$$

The few advantages of the Recursive Merging algorithm are its robustness to noisy data and missing value in the data set. This technique also has the ability to handle large global scale data set. Both sudden increase and decrease in vegetation are detected by this approach. The seasonality of data is taken into account by this algorithm, but it does not consider the variability present in the data.

The limitation of this approach is its inability to accurately identify a change point and detect multiple change points in time series. It also fails to explain the physical interpretation of the assigned change scores. This algorithm works only on temporal data and does not use spatial information that is present in the data set, although the earth science data sets exhibit significant spatio-temporal autocorrelation.

The computational complexity of the Recursive Merging algorithm is $\mathrm{O}(N t)$ (the cost of processing a single location is $t-12$ and there are $N$ locations). The storage requirement is $\mathrm{O}(N)[2,22]$.

\subsection{Yearly Delta algorithm}

Yearly Delta algorithm (YD) is a predictive-model-based approach and related to EWMA algorithm [24]. This algorithm was initially proposed by Boriah [15] and used by Mithal and his team [25, 26], which detects sudden changes in vegetation due to events like fires, floods, etc. YD uses a step function as the forgetting factor $(\lambda)$. Usually $\lambda$ specifies the relative level of importance attached between older observations and more recent observations. Here YD algorithm pays equal importance to both recent and older observation values. The algorithm predicts the expected EVI values for each time step of future years and then calculates the annual mean of the projected time series. The YD score for a location is assigned as the maximum difference between the annual mean of observed and projected model. The change time is decided based on a time step with the maximum score. The locations are arranged in descending order on the basis of YD score. Finally, certain numbers of the top ranked locations from the order list are considered as changes, because the locations disturbed by the event (fires or floods) unusually have a large EVI decrease from one year to the next. The time series for each location is processed by the Yearly Delta technique as follows.

Step 1. The model-based algorithm has a forgetting factor $\lambda$ and expected value $Z_{1}$. The expected value can be calculated by taking a short window (here initial 12 months) in observed time series.

$$
\begin{aligned}
& \lambda \leftarrow 0.5, \quad 0<\lambda \leq 1 \\
& Z_{1} \leftarrow \frac{\sum_{t=1}^{12} A_{t}}{12}
\end{aligned}
$$

where $A_{t}$ is EVI value at time step $t$ in observed time series. Here $\lambda$ is initialized as 0.5 , which provides equal importance to both recent and older observations.

Step 2. The projection model time series $Z$ is built using the $A_{t}$. Here, $A_{t}$ is the EVI value at time step $t$ present in observed time series; $t$ varies from 1 to $n-12$, where $n$ is the total number of time steps present in observed time series. The projection time series is calculated as follows:

$$
Z_{t+1} \leftarrow\left(\lambda A_{t+12}\right)+(1-\lambda) Z_{t} .
$$

Step 3. The annual mean of observed time series is calculated. Let $a_{j}$ be the annual mean and $j$ vary from 2 to $y$, such that, $a_{1}, a_{2}, \ldots, a_{y-1}$ are the annual mean present in the observed time series.

$$
a_{j-1} \leftarrow \frac{\sum A_{t-S+1, \ldots, t}}{12}
$$

where $A_{t}$ is the EVI value at time step $t$, and here $t=12 j$ and $S=12$.

Step 4. Similarly, annual mean of projection model time series is calculated. Let $P_{j}$ be the annual mean and $j$ vary from 1 to $y-1$, such that $P_{1}, P_{2}, \ldots, P_{y-1}$ are the annual mean present in the projection model time series.

$$
P_{j} \leftarrow \frac{\sum Z_{t-S+1, \ldots, t}}{12}
$$

where $A_{t}$ is the EVI value at time step $t$, and here $t=12 j$ and $S=12$.

Step 5. The deviation, $d_{j}$ is computed by taking the difference between annual mean of observed and projected model. Here, $j$ varies from 1 to $y-1$.

$$
d_{j} \leftarrow a_{j}-P_{j} .
$$

Step 6. The change score $C S$ is the maximum deviation observed.

$$
C S \leftarrow \max \left(d_{j}\right) .
$$

The YD technique is robust to missing values and scalable to large data sets. Also, it detects the time of change close to the actual change accurately, although to obtain the most accurate time estimation, post-processing may be required.

The restriction of the YD algorithm is its inability to detect multiple changes and changes in both directions. Furthermore, YD is sensitive to symmetric noise (noise that is not primarily skewed in the positive or negative direction). In particular, when changes are being detected in both 
directions, the scheme can confuse high scores due to tangible change or due to disturbance.

In YD algorithm, for $N$ location, the computational complexity is $\mathrm{O}(N t)$ and the storage requirement is $\mathrm{O}(N)[2]$.

\subsection{Persistent-DELTA algorithm}

Chamber and his team [27] proposed the Persistent-DELTA or PDELTA approach to identify the gradual vegetation change in time series data due to logging, insect infestation, reforestation, etc. The main objective of the PDELTA approach is to identify the window of maximum reliable drop in a time series. The drop may be continuous or may not be. In non-continuous drop, some amount of the intermittent rise can be allowed as long as a decreasing trend is persistent as shown in figure 2 . The reliability condition decides the permissible intermittent rise during an extended period of degradation. The approach is explained below in detail.

Initially compute the $\Delta_{i}$ by differencing two consecutive annual segments; mathematically $\Delta_{i}=V_{t_{i}-S+1, \ldots, t_{i}}$ $-V_{t_{i}+1, \ldots, t_{i}+S}$, where $V_{t_{i}}$ is value at time $t_{i}$ and $S$ is the number of time steps in a year. Then construct $\Delta_{i}$-series (Delta-series) by composing of $\Delta_{i}$ 's, $S \leq i \leq n-S$, where $n$ is the number of time steps in the time series. The resultant $\Delta_{i}$-series provides the drop relative to the previous year or trend information in a time series, which is more robust to seasonal variations and noisy outliers. Next compute a $\Gamma$ series (Gamma-series, which is scaled by a factor of 0.07) from $\Delta$-series, with each time step represented by $\Upsilon_{i}$, using the following transformation condition: if $\Delta_{i}>0$ then $\Upsilon_{i}=$ 1 and if $\Delta_{i} \leq 0$ then $\Upsilon_{i}=-1$. In the $\Gamma$-series, the $i$ th time steps are considered as candidates for drop start, for which
$\Upsilon_{i-1}$ is -1 and $\Upsilon_{i}$ is 1 (transition to 1 ) are denoted by $b_{k}$. Similarly, the $i$ th time steps are considered as candidates for drop end for which $\Upsilon_{i-1}$ is 1 and $\Upsilon_{i}$ is -1 (transition from 1) are denoted by $e_{k}$. Here all $k \in 1, \ldots, K$. Between every $b_{k}$ and $e_{k}$ there is a decrease in EVI values (decreasing trend), and between every $e_{k}$ and $b_{k+1}$ there is an increase in EVI values (increasing trend). If a drop starts at $b_{k}$, then an intermediate rise occurs between every $e_{l}$ and $b_{l+1}$ $(k \leq l<K)$. In order to ensure that the decreasing trend is followed across these intermediate rises, at every $e_{l}$ the reliability condition test must be satisfied.

The reliability condition states that after the commencement of a drop at a time step $b_{k}$, the rise occurring at a certain time step $e_{l}\left(e_{k} \leq e_{l}<e_{K}\right)$ would not be considered as drop termination, if this rise does not exceed $x \%$ of the drop that has already occurred. As soon as this condition fails, the drop that began at time step $b_{k}$ is terminated at $e_{l}$. This becomes a candidate drop window $\left(c w_{p}\right)$ for time series $T$. The same procedure is repeated for the remaining candidate start time steps in the same time series to find the other candidate drop windows $\left(c w_{p}\right)$, where all $p \in 1, \ldots, K$. Then compute a score for each candidate window using Length of the Drop Window or Total Loss in EVI. The maximum scoring window is determined as the representative window of the time series. The higher the score value, higher the degree of change. It is presented in figure 2 .

The approach is robust, scalable, identifies the periods of change and easy to apply across different regions and vegetation types. The main purpose of this approach is to detect gradual changes; hence, it may not detect sudden change in data. Different scoring mechanisms have been proposed for this algorithm, but none of the scores assign a statistical significance of the changes. The change events that recover very fast may not be detected as well.
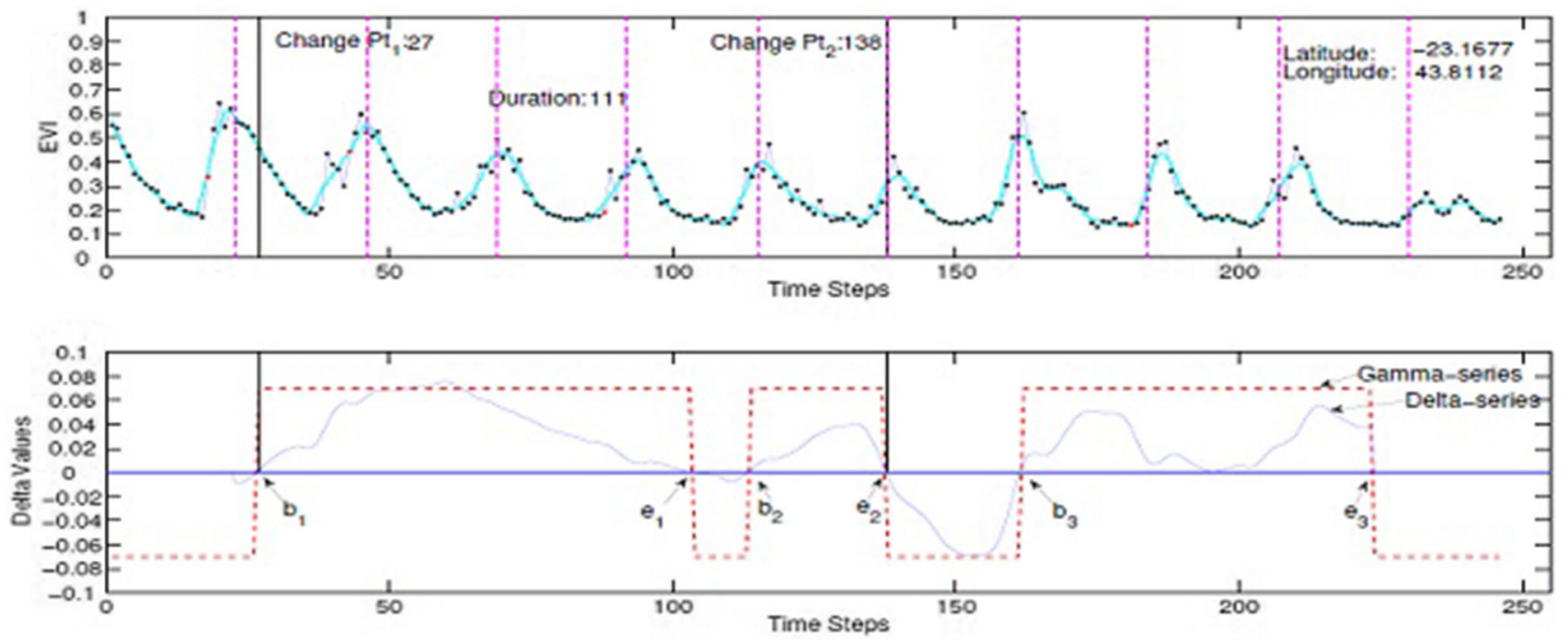

Figure 2. The top plot shows the original time series and the bottom plot shows the corresponding $\Delta$-series as well as the $\Gamma$-series scaled by a factor of 0.07 and the changed period (between vertical lines) [27]. 
For a grid with $M \times N$ observations and time-series length $T$, the time complexity of PDELTA is $\mathrm{O}(M N T)$ and the space requirements of this approach are modest [28].

\subsection{BFAST algorithm}

The Breaks for Additive Seasonal and Trend (BFAST) is a generic approach to detect long-term changes in satellite image time series, proposed by Verbesselt and his team [29]. This technique decomposes a time series data into trend, seasonal and remainder components, so that the intra-segment models are similar, while inter-segment models are dissimilar. A trend breakpoint is associated with segment boundaries. BFAST iteratively estimates the trend and seasonal components and allows for an individual estimation of breakpoints in the seasonal and the trend components. Sequential test methods for detecting breakpoints (i.e., abrupt changes) in a time series have been developed. These methods also allow linear models to be fitted to sections of a time series, with breakpoints at the times where the changes occur. The optimal position of these breaks can be determined by minimizing the residual sum of squares, and the optimal number of breaks can be determined by minimizing Bayesian Information Criterion (BIC). Before fitting the piecewise linear models and estimating the breakpoints, the ordinary least squares (OLS) residual-based MOving SUM (MOSUM) test is used to identify whether any breakpoints occur in the time series. As output, BFAST provides the trend breakpoints and associated trends, seasonal breakpoints and associated seasonal models, and logical values indicating whether the time series considered changes in the seasonal or trend components.

The strength of BFAST is robustness against noise and its ability to detect different types of changes such as gradual, abrupt and phenological change. It also estimates the date of change and finds multiple changes in time series. BFAST can be applied to other NDVI/EVI time series data such as hydrology, climatology and econometric data.

The major limitation of BFAST technique is positioning the segment boundaries at the time step where regression lines have best fit. This technique has high computational complexity; hence, it is not scalable for the global data set. Also, BFAST is unsuitable for highly variable time series, where noise levels can falsify the ideal seasonal pattern. BFAST also requires the minimum segment size and the maximum number of breakpoints though they are not mandatory. However, in the absence of these parameters, BFAST is quite sensitive to noise and as a result breaks even a single trend into multiple segments.

\subsection{Forest fire mapping algorithm}

Chen and his team [30] developed unsupervised spatiotemporal data mining methods for MODIS data to generate a history of forest fires. Forest fires are often described by a sudden decrease in the EVI time series and also responsible for emission of large amounts of thermal energy close to the land surface. Thus, the authors consider both the EVI and AF (Active Fire) data sets to identify fire events. AF is a basic fire product designed to identify thermal anomalies from the middle infrared spectral reflectance bands [31]. Forest fires are often characterized by a sudden decrease in the EVI time series and these drops may persist for a few years or for several months. Hence, authors defined three different scoring mechanisms: $K$-month Delta (KD), Local Instant Drop (LID) and Near Drop (ND). KD is designed to score the changes that persist for a long time ( $K$ months). It accounts for the natural variability present in the vegetation, which is specific to a particular region. LID scores the instant drop in EVI to identify fires that recover too quickly to be captured by the KD score. ND score reflects the real amount of drop in EVI when a fire happens. Pixels that are present in AF data set and satisfy one of these two scoring criteria, a high ND score with a large KD score or a high ND score with a large LID score, are considered as initial pixels (labelled as the highest stratum) for forest fire events. Generally the fire events corresponding to the same forest fire occur in close proximity of space and time. To increase coverage, they consider the 24 spatial neighbours in a $5 \times 5$ spatial grid around the initial pixels, with a temporal constraint of being within one time step from the change time of the initial fire event and apply the same scoring mechanism used for detecting initial forest fire events. The result representing forest fire events is labelled as the middle stratum. The included pixels in middle stratum have sharp fire characteristics of the EVI signal, but were not considered as initial pixels because of the absence in AF data set. The lowest stratum is generated by relaxing scoring criteria for similar events using a spatial window around the other two strata. The flow chart of fire mapping algorithm is shown in figure 3 .

The forest fire mapping algorithm is unsupervised in nature and has the potential to be used globally. Further, this algorithm is highly robust to noise because one of its primary inputs is AF data set.

The forest fire mapping algorithm may not work where the vegetation rapidly recovers after a fire, the loss in vegetation is insignificant or the vegetation has high natural variability, with multiple fires in short succession. If a fire polygon does not contain any pixel with an AF signal, then this algorithm fails to detect the fire event.

This algorithm takes $\mathrm{O}(t)$ time to process for a single location. Also, this algorithm takes $\mathrm{O}(M)$ time for searching in the AF data set to create the highest stratum. The total computational complexity of this algorithm for $N$ locations is $\mathrm{O}(M N t)$ and the storage requirement is $\mathrm{O}(N)$.

\subsection{Model-free segmentation algorithm}

Garg and his team [32] presented a novel model-free segmentation-based change detection algorithm for detecting land cover change in the EVI time series data. The locations 


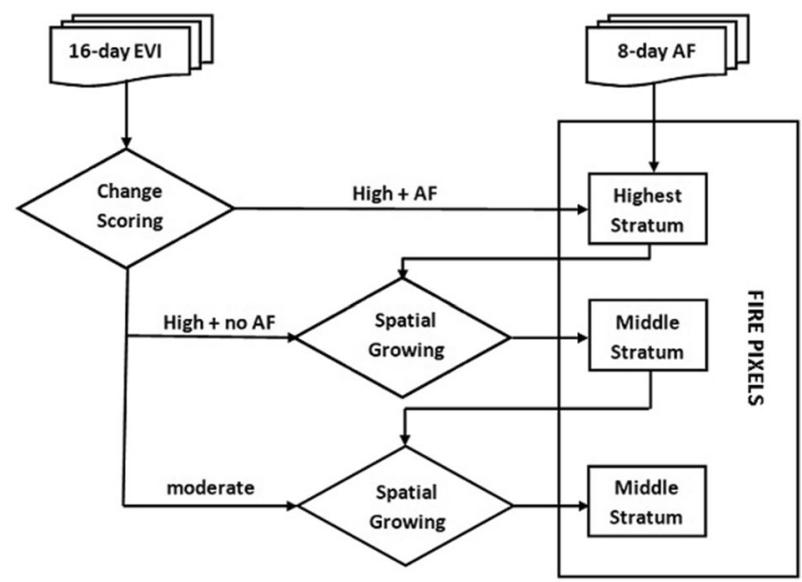

Figure 3. Flow chart for forest fires mapping [30].

with high change scores are more likely to have changed. The key idea of this algorithm is to find two segments in a given time series such that the intra-segment annual years are similar and inter-segment annual years are different. The similarity and differences between the annual years for each segment can be expressed by cohesion and separation, which is shown in figure 4 . The cohesion value of a segment is calculated by taking the average of pairwise distance between each annual year present within a segment. Cohesion $(C)$ for the time series of a pixel is determined by calculating the average of the cohesion value of both the segments. High cohesion value means the intra-segment year difference is high, which indicates high natural variability of the time series. The separation $(S)$ between two segments can be measured by the sum of the distance between each annual years present in one segment to annual years present in another segment. The separation value indicates to what extent the segments are apart from each other. The combination of cohesion and separation values indicates the amount of change in the time series with respect to the natural variation. The change score is the maximum of the difference between separation and cohesion value. The change point of time series is a time step

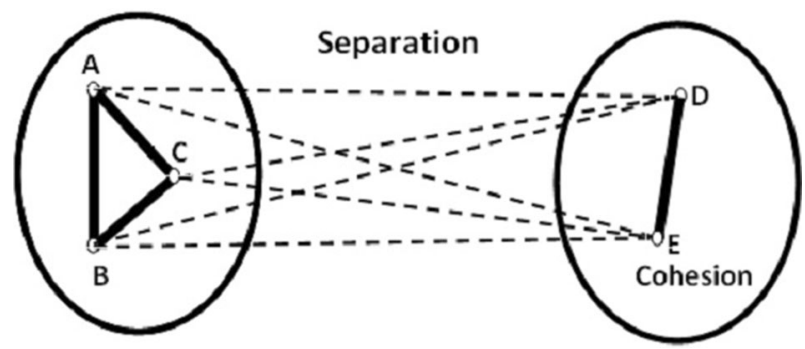

Figure 4. A-E represent the five different years in a time series. The two circles represent the two segments. The dark edges and dotted lines represent, respectively, the cohesion and separations between the segments [32]. where the change score is high. To maintain the inter-annual variability in each segment, the authors assume that the change points occur no earlier than the end of second year and no later than the second to last year. The algorithm processes the time series as follows.

Step 1. Calculate the Manhattan distance between each annual segment from other present in the time series $T$ and store in a dissimilarity matrix $M$.

Step 2. Calculate the cohesion value $C_{i}$ from segment 2 to $y-1$. Here $y$ is the total number of complete years present in time series $T$.

Step 3. Calculate the separation value $S_{i}$ from segment 2 to $y-1$. Here $y$ is the total number of complete years presents in time series $T$.

Step 4. Score $_{i}=S_{i}-C_{i}$.

Step 5. Repeat the steps 2-4.

Step 6. Change score $C S_{i}=\max \left(\right.$ Score $\left._{i}\right)$.

The key advantages of this algorithm are computational simplicity and efficiency. This algorithm takes into account the inherent variability present in the remote sensing data and can be applied globally for a variety of vegetation.

The main drawback of this algorithm is that it assumes that only one pattern change occurs in the time series. If the length of the time series is small, the calculations of cohesion and separation values are straightforward, but it becomes complicated as the length of the time series increases. Also, this technique assumes that the pattern changes occur at the yearly boundary, which is not always true in the land cover change domain.

This algorithm takes $\mathrm{O}(t)$ time to process for a single location. The total computational complexity of this algorithm for $N$ locations is $\mathrm{O}(N t)$ and the storage requirement is $\mathrm{O}(N)$.

\subsection{Recursive Search algorithm}

Panigrahi and his team [2] proposed the Recursive Search algorithm (RSA), which is a novel, simple and efficient segmentation-based land cover change detection technique. It detects the changes in time series and labels it with the change type, i.e., sudden increase or decrease type. RSA technique also detects the time of change depending on the temporal resolution (each 16 days or monthly) present in the time series. The central idea of the RSA technique is to get two consecutive annual segments in the time series such that the value at time step present within each annual segment is very similar while the value at time step present between the annual segment is significantly different. For this, the RSA technique first calculates the difference between each consecutive annual segment present in the time series using city-block distance, which exploits the seasonality of the time series data. The difference between consecutive annual segments shows how one segment is separated from the other. The RSA technique tries to find 
the consecutive annual segment difference with the maximum value and its index value by employing the two-way searching method. This algorithm processes the time series of each location as follows.

Step 1. Let $u$ and $v$ be the indices that, respectively, point to the first and last annual segment in time series. Then it finds the city-block distance between consecutive annual segments from the start and end of time series, which is stored in $d_{u}$ and $d_{v-1}$, respectively.

$$
\begin{aligned}
& d_{u} \leftarrow \sum\left(A_{(12 *(u-1)+1), \ldots,(12 * u)}-A_{((12 * u)+1), \ldots,(12 *(u+1))}\right) \\
& d_{v-1} \leftarrow \sum\left(A_{(12 *(v-2)+1), \ldots,(12 *(v-1))}-A_{((12 *(v-1)+1), \ldots,(12 * v))}\right) .
\end{aligned}
$$

Step 2. This technique compares the first consecutive annual segmentation difference value, $d_{u}$ to last consecutive annual segmentation difference value, $d_{v-1}$ and the largest difference value between the two is stored in another variable $S$ (initially $S=0$ ) and the corresponding index of difference array $(d)$ is stored in $j$.

Step 3. Repeat the steps 1 and 2 till it meets the stopping criteria, which is dependent on whether the total number of annual segments $y$ present in the time series is odd (i.e., stop condition is $(u>$ mid \& $v<$ mid $)$, here mid $=(y+1) / 2$ ) or even (i.e., stop condition is $(u \geq$ mid \& $v \leq$ mid $)$; here, mid $=y / 2$ ).

Step 4. From the resultant consecutive annual segments, the $j$ th and $(j+1)$ th elements represent unchanged and changed segment, respectively, which is shown in figure 5. In the time series data, 1st to jth annual segments follow a similar pattern, which is different from that of $(j+1)$ th annual segment.

$$
S_{\text {diff }} \leftarrow S_{\text {org }}-S_{\text {avg }}
$$

where $S_{\text {org }}$ is annual sum of EVI at $(j+1)$ th segment and $S_{\text {avg }}$ is annual sum of the month-wise EVI value average (avg) of 1 st to $j$ th segments.

Step 5. The change score (CScore) and change type (CT) for each location is computed from the value of $S_{\text {diff }}$ :

$$
C T \leftarrow\left\{\begin{array}{cc}
\text { CScore } \leftarrow \text { abs }\left(S_{\text {diff }}\right) \\
\text { if }\left(\mathrm{S}_{\text {diff }}>0\right) & \text { then }+ \text { ve type change, } \\
\text { elseif }\left(\mathrm{S}_{\text {diff }}<0\right) & \text { then }- \text { ve type change, } \\
\text { otherwise } & \text { nochange. }
\end{array}\right.
$$

Step 6. To determine the change point or time of change, the absolute values of $D_{j(j+1)}$ and $D_{(j+1)(j+2)}$ are concatenated and stored in $Y_{i}$.

$$
Y_{i} \leftarrow\left[a b s\left(D_{j(j+1)}\right), a b s\left(D_{(j+1)(j+2)}\right]\right.
$$

where $D_{j(j+1)}$ is month-wise difference between $j$ th and $(j+1)$ th annual segment. $D_{(j+1)(j+2)}$ is month-wise difference between $(j+1)$ th and $(j+2)$ th annual segment. Step 7. The authors assume that the change point lies before change segment; hence, they find the confidence of first 12 values of the $Y$. This can be evaluated by checking how many times an element $Y_{i}$ is greater than its next 12 elements such as $Y_{i+1}$ to $Y_{i+12}$. When the confidence percentage is greater than a certain user-defined threshold (Th) value, the point with the highest differential value (means highest $Y_{i}$ value) is designated as the change point.

The RSA technique finds sudden changes and identifies the single most significant change in a time series. This technique is robust to noise and scalable to large global scale data set. Also, this technique detects the time of change close to the actual change, which depends upon the temporal resolution of input data set.

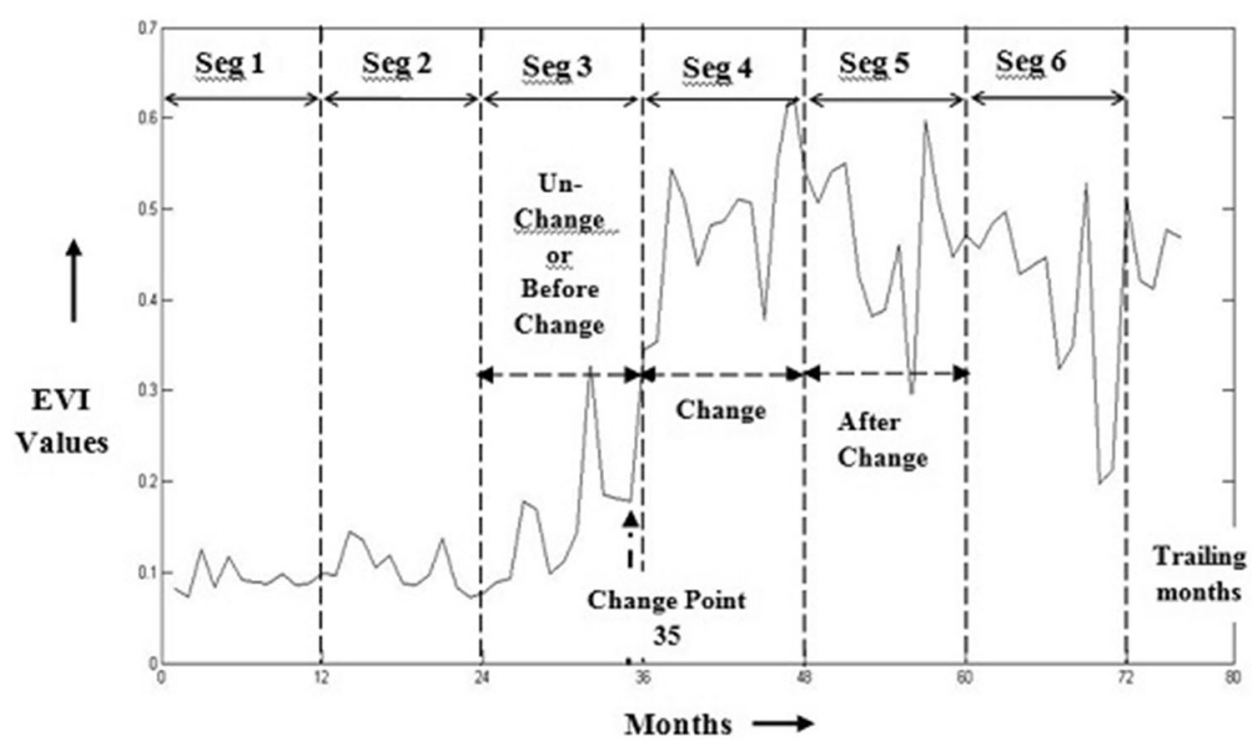

Figure 5. The RSA algorithm determines the change segment as well as change point [2]. 
The RSA algorithm fails to detect any change if a change event occurs during the first and last year of the time series data. This method assumes and finds the change point before the change segment, which may not be correct always, because the change point may occur at the initial time of the change segment.

The cost of processing by RSA algorithm for a single location is $t$. There are $N$ locations; hence, the time complexity of RSA is $\mathrm{O}(N t)$. The storage requirements is $\mathrm{O}(N)[2]$.

\section{Empirical study}

We have used the quantitative evaluation method to study and realize the comparative performance of a few existing land cover change detection algorithms. Here we have used EVI data sets (MOD13Q1) of location, latitude $32.15940493^{\circ}$ to $37.43440247^{\circ}$ and longitude $-122.3595558^{\circ}$ to $-114.9427576^{\circ}$, from periods February 2000 to September 2015, which is downloaded from LP DAAC [Available: https://www.lpdaac.usgs.gov/products/ modis_products_table].

Data pre-processing is performed by the following steps:

1. The biweekly data are converted to monthly data by averaging (using a simple mean) the available data for every month.

2. The MODIS data sets are tagged with a quality assurance (QA) flag, which describes the atmospheric and sensor conditions in which the measurements are taken. All the measurements that are tagged to low quality are removed.

3. Another filtering is performed by removing the EVI value less than 0.1 and above 0.9 , suggested by earth science domain experts [23].

4. Then we discarded the locations that contain any missing data. As a result, the data for a location is retained only if the entire time series is available without any missing values and no low quality data.

5. Furthermore, from the resultant data set, we have selected manually 750 time series data for our experiments and validation purposes, which is labeled as DS. The DS data set consists of three different types, namely: 600 normal, 75 sudden increasing and 75 sudden decreasing time series data. A sample of each type of time series present in the DS data set is shown in figure 6 .

In the DS data set, the length of the time series is 188 , which corresponds to 15 years and 8 months of monthly data from February 2000 through September 2015. Here, each value present in the time series corresponds to EVI value of particular months. The normal type time series are labelled as the unchanged class, whereas both sudden increase and sudden decrease time series are labelled as changed class.
We used the standard evaluation metrics, i.e., precision, recall, $F$-score and accuracy, to study the relative performance of land cover change detection algorithms, because these metrics are often employed to measure the performance of algorithms in information retrieval, machine learning and data mining [33, 34].

Let $D$ be a data set with $N$ time series and each time series correspond to an exact location. The data set also consists of true labels for each location, i.e., changed and unchanged labels. Let $M$ be the genuine numbers of changed labels (sudden increase and decrease time series) present in the data set, called as validation data. For each location, the change detection algorithm computes and assigns a score. Finally, the algorithm returns a list of $N$ change scores corresponding to $N$ locations and each change score represents the degree of change at a particular location. Subsequently, the locations are ordered in the descending order of their change score. The top $n$ (normally $1 \leq n \leq M)$ ranked locations present in the list are considered as change events and others are conceived as unchanged. By counting the overlap with the validation data, we work out true positives $\left(T P_{n}\right)$, changes detected by the scheme also present in the validation data; false positives $\left(F P_{n}\right)$, changes found by the scheme but not in the validation data; true negatives $\left(T N_{n}\right)$, changes not found by the scheme and also not present in the validation data and false negatives $\left(F N_{n}\right)$, changes noted in the validation data but not found by the scheme, for each algorithm. The precision, recall, $F$-score and accuracy can be defined mathematically as follows:

$$
\begin{aligned}
& \text { precision }\left(p_{n}\right)=\frac{T P_{n}}{T P_{n}+F P_{n}}, \\
& \text { recall }\left(r_{n}\right)=\frac{T P_{n}}{M}, \text { where } M=T P_{n}+F N_{n}, \\
& F \text {-Score }(F)=2 \times\left(\frac{\text { Precision } \times \text { Recall }}{\text { Precision }+ \text { Recall }}\right), \\
& \text { accuracy }=\frac{T P_{n}+T N_{n}}{T P_{n}+F P_{n}+T N_{n}+F N_{n}} .
\end{aligned}
$$

Precision is a measure of quality, whereas recall is a measure of quantity. $F$-score is a measure of a test's accuracy. Accuracy presents the overall correctness of the model.

\subsection{Empirical results and discussion}

The EVI time series data set DS is input to the Modified Lunetta, CUSUM MEAN, Yearly Delta, Recursive Merging and RSA algorithms. Each of the algorithms returned output as a list of change scores. The afore-discussed algorithms consider that among the great coverage of ground cover data, only a small region exhibits change. A location is conceived as changed, when the change score of that particular location is greater than some threshold value. 


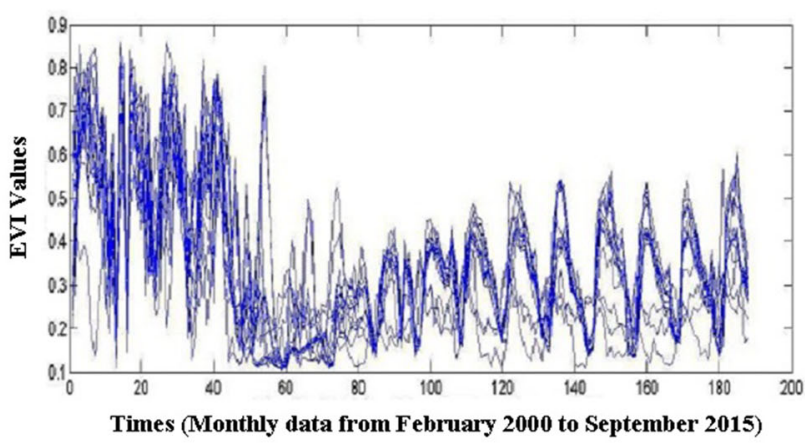

(a) Sudden decrease time series

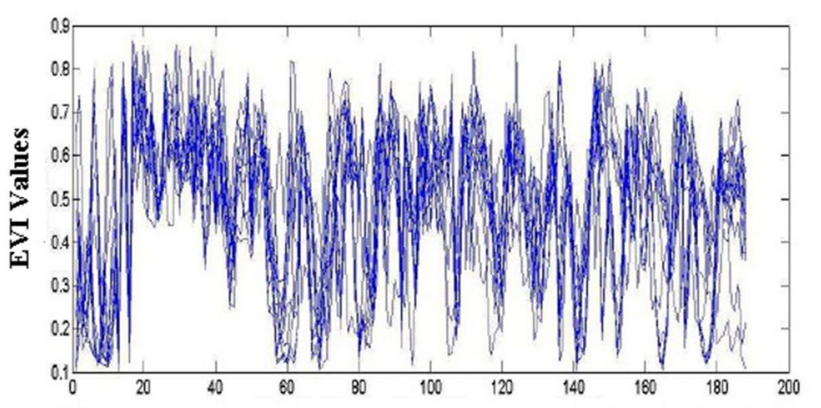

Times (Monthly data from February 2000 to September 2015)

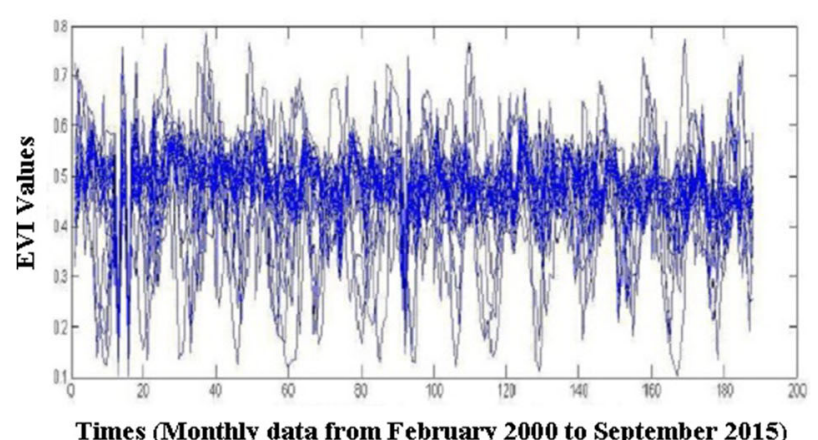

(c) Normal time series

Figure 6. Example of three types of time series data present in DS data set.

The threshold value has been determined from the histogram by selecting the score, when the change score frequency suddenly reduces. Figure 7 shows the histogram of the change score obtained by RSA. From figure 7, the threshold value for RSA is observed to be 22.5. Likewise, we set the threshold value for the Modified Lunetta, CUSUM MEAN, Yearly Delta and Recursive Merging algorithms, which are $25,23,26$ and 39.5, respectively, for the DS data set. The validation result and performance of the algorithms are presented in table 2 .

Table 2 demonstrates the evaluation result of the DS data set. The Modified Lunetta gives $25.2 \%$ wrong result, causing very poor precision and recall, and gives an accuracy of $74.8 \%$. The CUSUM MEAN and Yearly Delta methods contain the same false negatives; as a result, both algorithms provide quite similar recall values. However, acceptable false positive of CUSUM MEAN algorithm produced good precision percentages as compared with the Yearly Delta algorithm. The accuracy of CUSUM MEAN and Yearly Delta methods are $82.93 \%$ and $78.8 \%$, respectively. The Recursive Merging and RSA have $19.3 \%$ and $4 \%$ false negative, respectively; hence, they have very good precision and recall values. The Recursive Merging and
RSA provide an accuracy of $91.6 \%$ and $99.2 \%$, respectively. Figure 8 shows a comparison of precision, recall, $F$ score and accuracy of each discussed algorithm.

The algorithms were implemented through MATLABR2014a and the codes were executed on a desktop with the following specifications: Intel(R) Core(TM) i5-4570M CPU @3.2GHz, 8GB of RAM, 64-bit Microsoft Windows 8 operating system. For calculation of elapsed time, the experiments were repeated ten times and the results averaged as presented in table 2 . The elapsed time comparisons of afore-discussed algorithms are shown in figure 9.

Figure 9 shows that the elapsed times of Modified Lunetta, CUSUM MEAN, Yearly Delta and RSA are very less as compared with that of Recursive Merging. The elapsed time of Yearly Delta is greater than that of Modified Lunetta and CUSUM MEAN, because it uses time for computation of the prediction model. In the analysis, we found that the elapsed time of the CUSUM MEAN algorithm is less, due to its computational simplicity and ease of implementation. The Modified Lunetta method elapsed time is a little more due to more computation, i.e., calculation of $z$-score of the difference of annual sums. The Recursive Merging technique spends more time on 


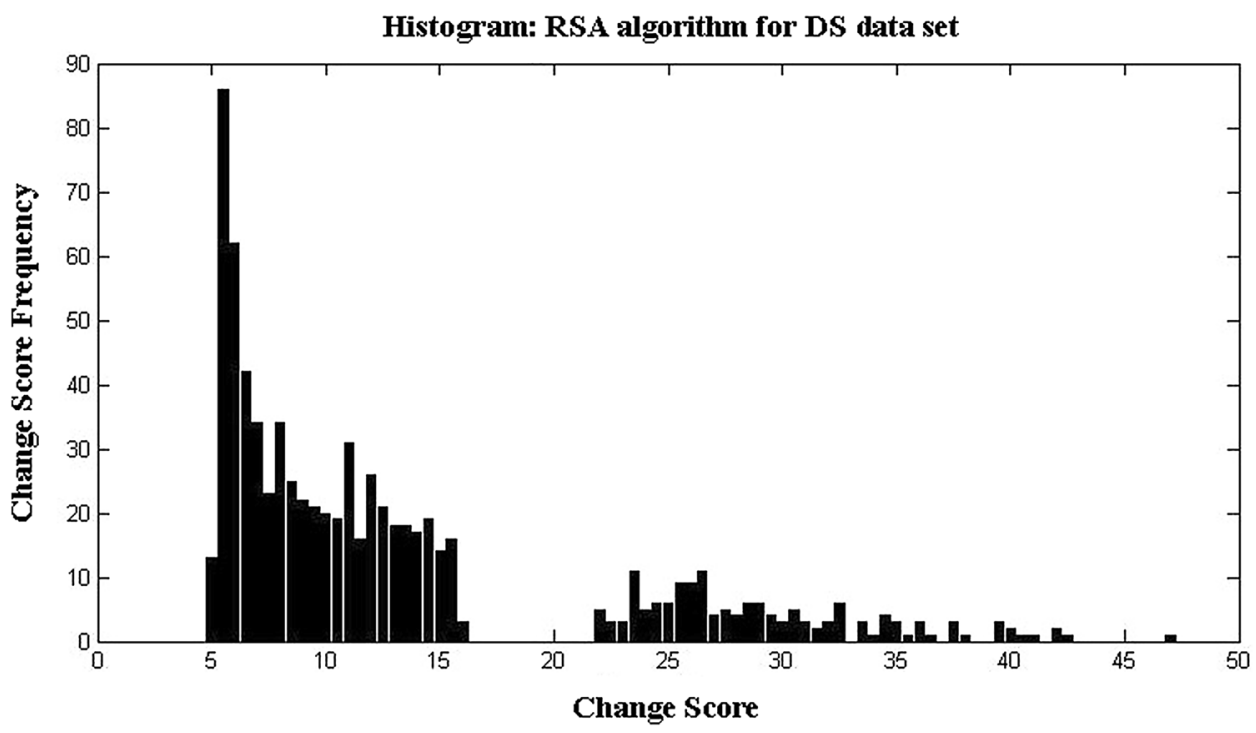

Figure 7. Histogram of change scores produced by the Recursive Search algorithm for DS data set.

Table 2. Evaluation result of DS data set.

\begin{tabular}{llrrrrrrrrr}
\hline Sl. no. & \multicolumn{1}{c}{ Technique } & $T P_{n}$ & $F N_{n}$ & $T N_{n}$ & $F P_{n}$ & Precision & Recall & $F$-score & Accuracy & Elapsed time (s) \\
\hline 1 & Modified Lunetta algorithm & 46 & 104 & 515 & 85 & 0.3511 & 0.3067 & 0.3274 & 0.748 & 0.9991608 \\
2 & CUMSUM MEAN algorithm & 65 & 85 & 557 & 43 & 0.6019 & 0.4333 & 0.5039 & 0.8293 & 0.6699521 \\
3 & Yearly Delta algorithm & 65 & 85 & 526 & 74 & 0.4676 & 0.43333 & 0.4498 & 0.788 & 1.2107509 \\
4 & Recursive Merging algorithm & 121 & 29 & 566 & 34 & 0.7806 & 0.8067 & 0.7934 & 0.916 & 6.7670502 \\
5 & Recursive Search algorithm & 144 & 6 & 600 & 0 & 1 & 0.96 & 0.9796 & 0.992 & 1.9187361 \\
\hline
\end{tabular}

\section{Data Set: DS}

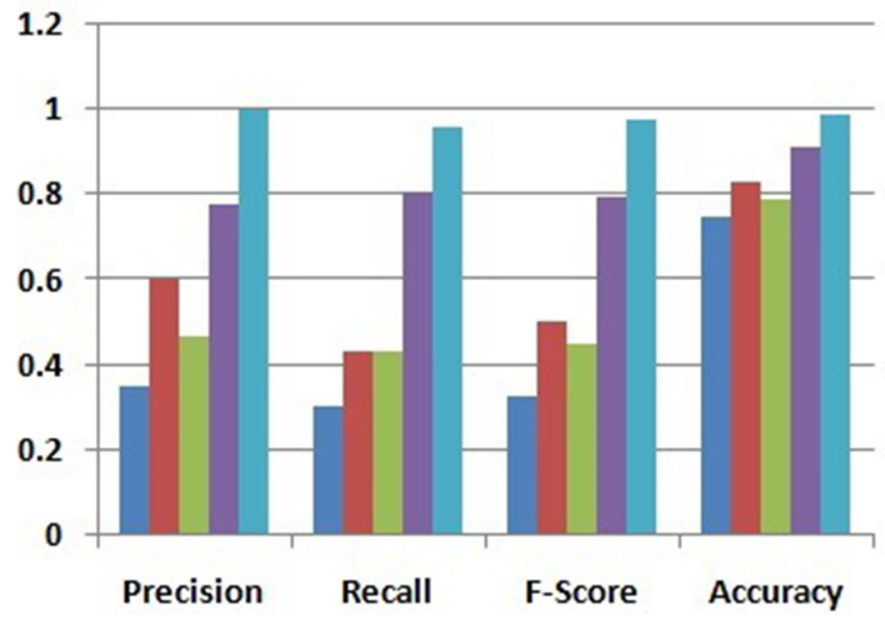

Modified Lunetta

CUSUM MEAN

Yearly Delta

Recursive Merging

Recursive Search

Algorithm

Figure 8. Performance comparison of algorithms on DS data set.

searching similar annual segments in time series data; hence, its elapsed time is more prominent than those of other methods. The elapsed time for RSA is less than that of the Recursive Merging, because RSA implements two-way indexing technique to search the highest segment difference in time series data, which reduces computation time. Also, 


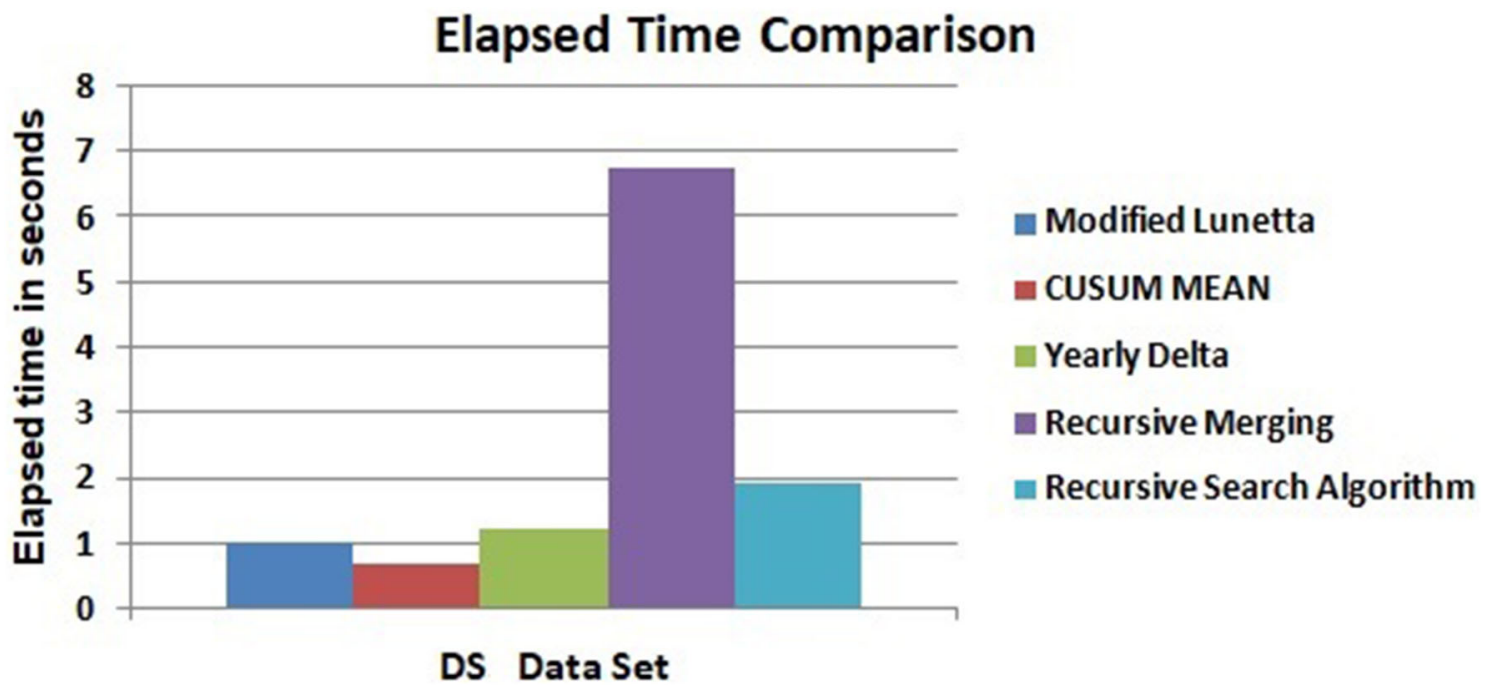

Figure 9. Comparison of elapsed time of algorithms on DS data set.

elapsed time of RSA is higher than that of other methods, because it not only provides the change score but also detects the change types and change time.

\section{Discussion}

All the afore-discussed data mining approaches are capable of handling massive data sets [2, 9, 15, 16, 22, 23, 27, 30, 32], which is one of the key requirements for global scale land cover change studies. CUSUM MEAN primarily detects changes in one direction, but by the distinctive characteristics of this technique it is possible to find both small and gradual shifts. The Lunetta technique finds changes in both directions (means sudden increase and reduction). The original Lunetta algorithm utilizes the spatial information present in the data set, but the Lunetta Mean algorithm does not consider the spatial information. The CUSUM MEAN algorithm performs better than Lunetta Mean and Yearly Delta. Recursive Merging is likely to outperform in some aspects than the Yearly Delta and CUSUM MEAN algorithms [23]. CUSUM MEAN and Lunetta technique fail to address noise and missing values, but the Yearly Delta algorithm performs well in the presence of missing values whereas it is sensitive to noise. The Recursive Merging algorithm and RSA are robust to noisy data and scalable to large data sets. Both the approaches account for seasonality and variability in the time series data, but in the presence of cyclic data, RSA technique substantially outperforms [2]. Recursive Merging detects change points at the boundaries between the segments, which may or may not be accurate, whereas RSA determines the change point close to the actual change with accuracy up to the temporal resolution using a confidence test. The clustering techniques first discover the dominant land cover patterns present in the data and then characterize the changes in terms of clusters. Here, $k$-mean clustering technique is used, but it is vulnerable to noisy data. PDELTA is more robust in the presence of noise and spurious changes. It detects changes that occur at a location by gradually decreasing vegetation and it is also able to quantify the magnitude of change in a time series. PDELTA has the ability to identify a gradual increase in vegetation, i.e., reforested area. The gradual increase in vegetation is determined by just simply reversing VI time series before applying the PDELTA algorithm [27]. BFAST, a generic approach, enables detection of gradual, abrupt and phenological changes in time series. It iteratively estimates the dates and number of changes in time series. BFAST is robust against noise, and is not influenced by changes in amplitude of the seasonal component. PDELTA performs substantially better than BFAST [27]. The forest fire mapping algorithm is an unsupervised spatio-temporal data mining method that detects land cover change due to forest fires and has the potential to be used globally. The forest fire mapping framework is highly robust to noise because one of its primary input is AF data set from MODIS. The model-free segmentation algorithm has the ability to provide rapid, inexpensive, robust, scalable and precise detection of land use change. This algorithm can be applied globally and is able to detect single-change pattern such as gradual or abrupt drops in the time series. It does not address noise and missing values. A summary of aforediscussed land cover change detection algorithms is provided in table 3. In table 3, $f(n), S, N, T$ and $t$ represent time complexity, space complexity, number of land locations, number of time steps in time series and total number of months present in the $y$ complete years, respectively. 


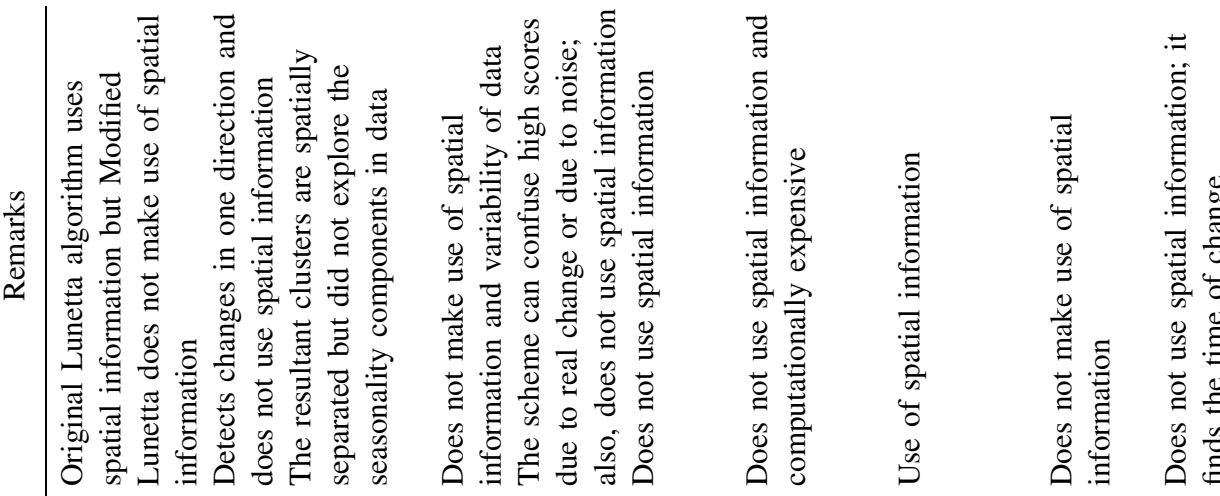

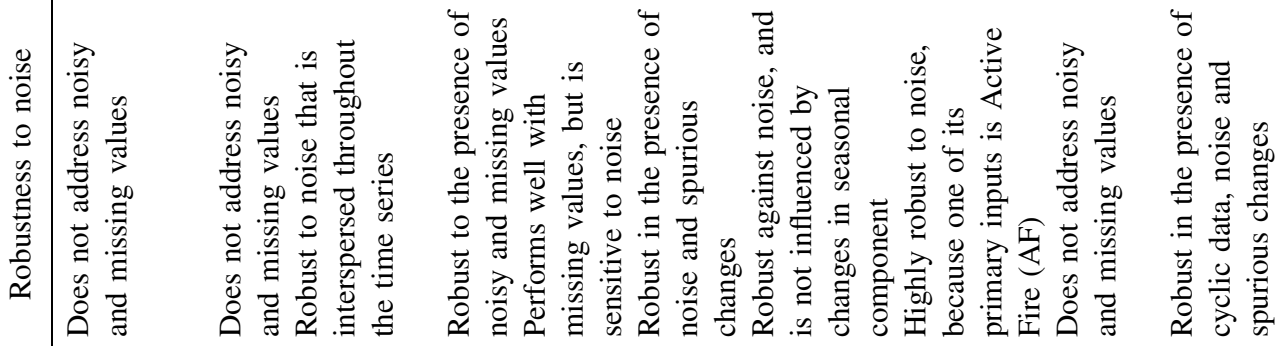

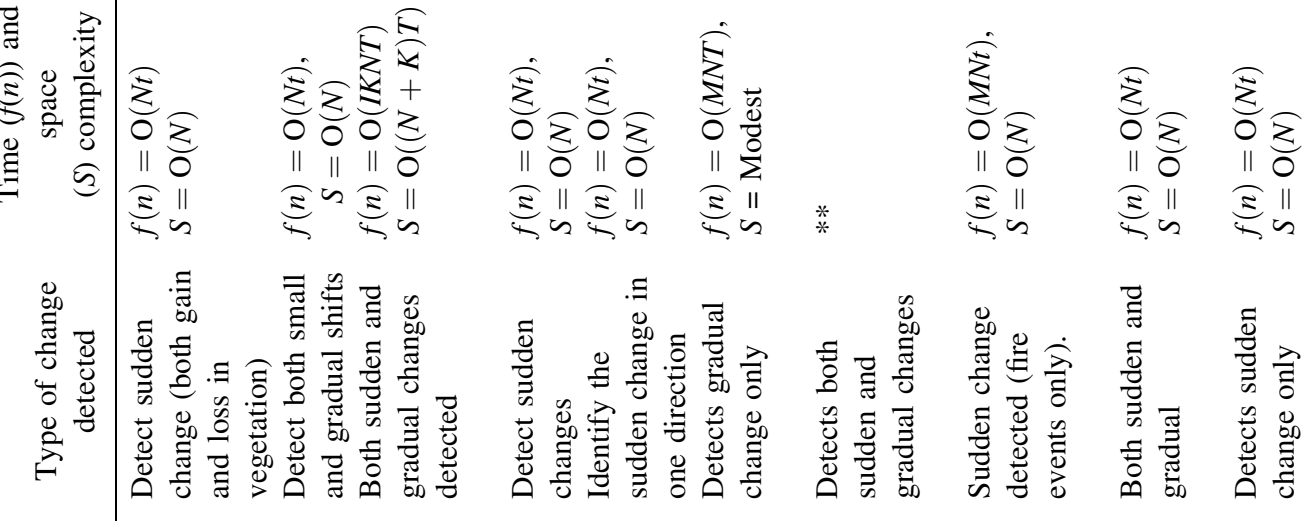

है

芩
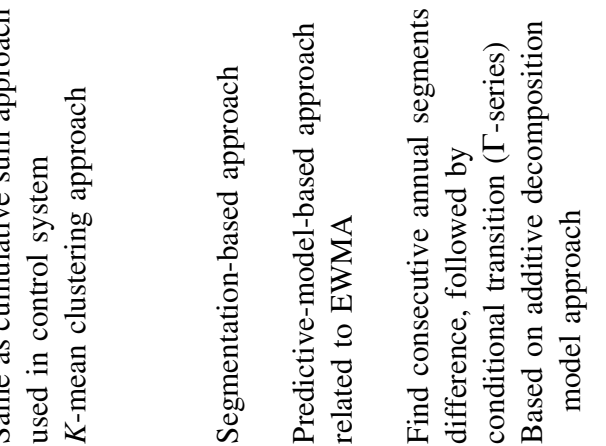

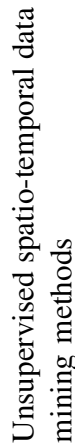
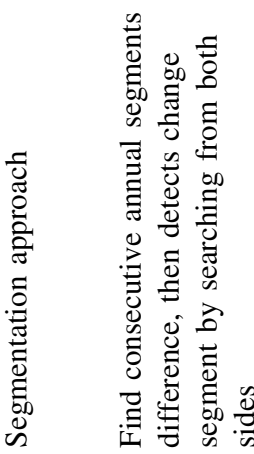


\section{Conclusion}

In this paper, we have reviewed the most popular data mining algorithms, which detect the land cover change by detecting the sudden change and/or gradual change in the vegetation index time series data set. Most of the algorithms have been implemented [2] and the advantages and limitations have been experimentally observed. An empirical study of some existing land cover change detection algorithms and results has been presented in this paper. The empirical study shows that the RSA technique performs better than Modified Lunetta, CUSUM MEAN, Yearly Delta and Recursive Merging algorithms. The data mining technique has emerged as an ultimate data-driven technique [35] to find the change detection in EVI/NDVI time series data because it is scalable, robust to noise and missing data and has nominal computational requirements $[2,9,15,16,22,23,27,30,32]$. The spatial information present in the EVI data set has not been used in many of the afore-discussed data mining techniques. Earth Science data sets exhibit significant spatio-temporal auto-correlation $[22,36,37]$, i.e., measured values that are close in time and space tend to be highly correlated, or similar. The use of spatial information can be expected to give better performance than existing data mining techniques [38, 39]. In the future works, we will propose a data mining approach for land cover change detection utilizing the spatial information present in vegetation index time series data.

\section{References}

[1] Coppin P, Jonckheere I, Nackaerts K, Muys B and Lambin E 2004 Digital change detection methods in ecosystem monitoring: a review. Int. J. Remote Sens. 25(9): 1565-1596

[2] Panigrahi S, Verma K and Tripathi P 2016 An efficient approach to detect sudden changes in vegetation index time series for land change detection. IETE Tech. Rev. 33(5): $539-556$

[3] Nemmour H and Chibani Y 2010 Support vector machines for automatic multi-class change detection in Algerian capital using Landsat TM imagery. J. Indian Soc. Remote Sens. 38(4): 585-591

[4] Singh K K, Nigam M J, Pal K and Mehrotra A 2014 A fuzzy Kohonen local information C-Means clustering for remote sensing imagery. IETE Tech. Rev. 31(1): 75-81

[5] Potter C, Tan P N, Kumar V, Kucharik C, Klooster S, Genovese V, Cohen W and Healey S 2005 Recent history of large-scale ecosystem disturbances in North America derived from the AVHRR satellite record. Ecosystems 8(7): 808-824

[6] Roy D P, Lewis P E and Justice C O 2002 Burned area mapping using multitemporal moderate spatial resolution data: a bi-directional reflectance model-based expectation approach. Remote Sens. Environ. 83(12): 263-286

[7] Huete A R, Justice C and Leeuwen W V 1999 MODIS vegetation index (MOD13) algorithm theoretical basis document. Version 3, Department of Environmental Sciences, University of Virginia

[8] Panigrahi S, Verma K and Tripathi P 2015 Review of MODIS EVI and NDVI data for data mining applications, communicated

[9] Lunetta R S, Knight J F, Ediriwickrema J, Lyon J G and Worthy L D 2006 Land-cover change detection using multi-temporal MODIS NDVI data. Remote Sens. Environ. 105(2): 142-154

[10] Keogh E, Chu S, Hart D and Pazzani M 2004 Segmenting time series: a survey and novel approach. In: Last M, Kandel A and Bunke H (Eds) Data mining in time series databases, vol. 57, pp. 1-22. Singapore: World Scientific

[11] Boriah S, Kumar V, Potter C, Steinbach M and Klooster S 2008 Land cover change detection using data mining techniques. TR 08-009, pp. 1-16

[12] Page E S 1954 Continuous inspection schemes. Biometrika 41(1-2): 100-115

[13] Kucera J, Barbosa P and Strobl P 2007 Cumulative sum charts: a novel technique for processing daily time series of modis data for burnt area mapping in Portugal. In: Proceedings of IEEE MultiTemp 2007, pp. 1-6

[14] Taylor W A 2000 Change-point analysis: a powerful new tool for detecting changes. http://www.variation.com/cpa/ tech/changepoint.html

[15] Boriah S 2010 Time series change detection: algorithms for land cover change. PhD Thesis, Department of CSE, University of Minnesota

[16] Boriah S, Kumar V, Steinbach M, Tan P N, Potter C and Klooster S 2008 Detecting ecosystem disturbances and land cover change using data mining. In: Next generation of data mining. CRC Press. Chapter 2, pp. 29-46

[17] Potter C, Genovese V, Gross P, Boriah S, Steinbach M and Kumar V 2007 Revealing land cover change in California with satellite data. EOS Trans. Am. Geogr. Union 88(26): 269-276

[18] Jain A K and Dubes R C 1988 Algorithm for clustering data. In: Prentice Hall advanced reference series. Prentice Hall. http://www.cse.msu.edu/ jain/Clustering_Jain_Dubes.pdf

[19] Salmon B P, Olivier J C, Wessels K J, Kleynhans W, Bergh F and Steenkamp K C 2011 Unsupervised land cover change detection: meaningful sequential time series analysis. IEEE J. Sel. Top. Appl. Earth Observ. Remote Sens. 4(2): 327-335, https://doi.org/10.1109/JSTARS.2010.2053918

[20] Ward J 1963 Hierarchical grouping to optimize an objective function. J. Am. Statist. Assoc. 58(301): 236-244

[21] Mitchell T 1997 Machine learning. New York: McGraw Hill

[22] Boriah S, Kumar V, Steinbach M, Potter C and Klooster S 2008 Land cover change detection: a case study. In: Proceedings of the 14th ACM SIGKDD International Conference on Knowledge Discovery and Data Mining, KDD 08, pp. 857-865

[23] Boriah S, Mithal V, Garg A, Kumar V, Steinbach M, Potter $\mathrm{C}$ and Klooster S 2010 A comparative study of algorithms for land cover change. In: Proceedings of the 2010 Conference on Intelligent Data Understanding, pp. 175-187

[24] Lucas J M and Saccucci M S 1990 Exponentially weighted moving average control schemes: properties and enhancements. Technometrics 32(1): 1-12

[25] Mithal V, Garg A, Boriah S, Steinbach M, Kumar V, Potter C, Klooste S and Castilla-Rubio J C 2011 Monitoring global forest cover using data mining. ACM Trans. Intell. Syst. Technol. 2(4): 36 
[26] Mithal V, Garg A, Brugere I, Boriah S, Kumar V, Steinbach M, Potter C and Klooste S 2011 Incorporating natural variation into time series based land cover change detection. In: Proceedings of the Conference on Intelligent Data Understanding, pp. 45-59

[27] Chamber Y, Mithal V, Garg A, Brugere I, Lau M, Krishna V, Boriah S, Potter C and Klooster S 2011 A novel time series based approach to detect gradual vegetation changes in forests. In: Proceedings of the 2011 Conference on Intelligent Data Understanding, pp. 248-262

[28] Faghmous J H, Chamber Y, Boriah S, Vikeb F, Liess S, Mesquita M and Kumar V 2012 A novel and scalable spatiotemporal technique for ocean eddy monitoring. In: Proceedings of the Twenty-Sixth AAAI Conference on Artificial Intelligence, $A A A I$ 12, pp. 281-287

[29] Verbesselt J, Hyndman R, Newnham G and Culvenor D 2010 Detecting trend and seasonal changes in satellite image time series. Remote Sens. Environ. 114(1): 106-115

[30] Chen X C, Karpatne A, Chamber Y, Mithal V, Lau M, Steinhaeuser K, Boriah S, Steinbach M, Kumar V, Potter C, Klooster S A, Abraham T, Stanley J D and Castilla-Rubio J C 2012 A new data mining framework for forest fire mapping. In: Proceedings of the Conference on Intelligent Data Understanding, CIDU' 12, pp. 104-111

[31] Justice C O, Giglio L and Roy D 2011 MODIS-derived global fire products. In: Ramachandran B, Justice C O and Abrams M J (Eds) Land remote sensing and global environmental change. Berlin: Springer, pp. 661-679
[32] Garg A, Manikonda L, Kumar S, Krishna V, Boriah S, Steinbach M, Toshnival D, Kumar V, Potter C and Klooster S A 2011 A model-free time series segmentation approach for land cover change detection. In: Proceedings of CIDU'11, pp. 144-158

[33] Tan P N, Steinbach M and Kumar V 2006 Introduction to data mining. Cambridge: Addison-Wesley Longman, p. 769

[34] Han J, Kamber M and Pei J 2011 Data mining: concepts and techniques, 3rd ed. Morgan Kaufmann, p. 744, ISBN:01238 14790

[35] Chen M S, Han J and Yu P S 1996 Data mining: an overview from a database perspective. IEEE Trans. Knowl. Data Eng. 8(6): 866-883

[36] Tan P, Steinbach M, Kumar V, Potter C, Klooster S and Torregrosa A 2001 Finding spatio-temporal patterns in earth science data. In: Proceedings of the KDD 2001 Workshop on Temporal Data Mining, vol. 19, pp. 1-12

[37] Steinbach M, Tan P N, Kumar V, Potter C, Klooster S and Torregrosa A 2001 Clustering earth science data: Goals, issues and results. In: Proceedings of the Fourth KDD Workshop on Mining Scientific Datasets, pp. 1-8

[38] Shekhar S, Zhang P and Huang Y 2009 Spatial data mining. In: Data mining and knowledge discovery handbook. USA: Springer, pp. 837-854

[39] Bogorny V and Shekhar S 2010 Spatial and spatio-temporal data mining. In: Proceedings of the 10th IEEE International Conference on Data Mining (ICDM), pp. 1217-1217 